\title{
Effect of the Distribution of Substitution of the Sodium Salt of Carboxymethylcellulose on Its Absorbency toward Aqueous Liquid
}

\author{
Kenji Kamide, Kunihiko OKaJima, Keisuke KowsaKa, \\ Toshihiko MATSUI, Sigeru NomURA, and Kunio HIKICHI* \\ Textile Research Laboratory, Asahi Chemical Industry Co., Ltd., \\ 11-7 Hacchonawate, Takatsuki, Osaka 569, Japan \\ *Department of Polymer Science, Faculty of Science, \\ Hokkaido University, Kita-ku, Sapporo 060, Japan
}

(Received November 9, 1984)

\begin{abstract}
An attempt was made to correlate the capacity of the aqueous liquid absorption of sodium salt of carboxymethylcellulose with its chemical structure, in particular with the total degree of substitution $\langle F\rangle$ and distribution of substitution $\left\langle\mathrm{f}_{k}\right\rangle$. Here, $\left\langle\mathrm{f}_{k}\right\rangle$ is the average degree of substitution of hydroxyl group attached to $\mathrm{C}_{k}$ carbon $(k=2,3$, and 6$)$ in an anhydroglucopyranose unit. For this purpose, 26 samples of $\mathrm{NaCMC}$ having $\langle\mathrm{F}\rangle$ less than 0.64 were synthesized by reacting cellulose having crystal form of cellulose I or II with a mixture of sodium hydroxide, monochloroacetic acid and 2-propanol. Their absorbency was determined at $37^{\circ} \mathrm{C}$ toward water, $0.9 \mathrm{wt} \%$ aq. sodium chloride, calcium chloride and aluminium chloride solutions. $\left\langle\mathrm{f}_{2}\right\rangle+\left\langle\mathrm{f}_{3}\right\rangle$, $\left\langle\mathrm{f}_{6}\right\rangle$, and $\langle\mathrm{F}\rangle$ were determined for these polymers by ${ }^{13} \mathrm{C}$ NMR in $10 \mathrm{wt} \%$ sodium hydroxide$90 \mathrm{wt} \% \mathrm{D}_{2} \mathrm{O}$ mixture. On using the cellulose with the crystal form cellulose II, carboxymethylation occurred almost preferentially at the hydroxyl group attached to the $\mathrm{C}_{6}$ carbon. This was not observed for the cellulose having the crystal form of cellulose I. Absorbency was found to be definitely governed by $\left\langle\mathrm{f}_{6}\right\rangle$, which can be explained in consideration of the solid structure of cellulose and $\mathrm{NaCMC}$.
\end{abstract}

KEY WORDS Sodium Salt of Carboxymethylcellulose / Distribution of Degree of Substitution / Absorbency / Cellulose I / Cellulose II /

Jansen $^{1}$ synthesized carboxymethylcellulose (CMC) as early as in 1918 using the reaction of alkali cellulose with sodium monochloroacetate. Since then, CMC with a total degree of substitution $\langle\mathrm{F}\rangle$ of $0.5-1.0$ has been commercialized world-wide and found numerous applications as stabilizing, thickening and absorbing agents in printing, detergent, foodstuffs, medicine, toilet, sanitary and petroleum industries. At the present, the industrial manufacturing of CMC is carried out either in aqueous or organic media. ${ }^{2,3}$ Natural cellulose with the crystal form of cellulose I (hereafter simply referred to as cellulose I) is exclusively used in the present commercial process, but no regenerated cellulose with the crystal form of cellulose II (hereafter referred to as cellulose II) is used.

Characteristic features, including solubility in water and aqueous salt solutions and interaction with cationic compounds, have been discussed so far only in a non-systematic manner. ${ }^{4,5}$ Recently, Kamide and his coworkers ${ }^{6}$ found the anticoagulant activity and acute toxicity of the sodium salt of cellulose sulfate to be quite closely related to its molecular properties, and in particularly to the probability of the substitution of hydroxyl groups attached to the $\mathrm{C}_{2}, \mathrm{C}_{3}$, and $\mathrm{C}_{6}$ carbons in the glucopyranose unit, $\left\langle\mathrm{f}_{2}\right\rangle,\left\langle\mathrm{f}_{3}\right\rangle$, and $\left.\left\langle\mathrm{f}_{6}\right\rangle\right\rangle$. The anticoagulant activity increased strongly with an increase in $\left\langle\left\langle f_{2}\right\rangle+\left\langle\left\langle f_{3}\right\rangle\right.\right.$. The chemical and 


\section{K. KAMIDE et al.}

physical properties of cellulose derivatives, including $\mathrm{CMC}$, should thus be governed primarily by $\left.\left\langle\mathrm{f}_{k}\right\rangle\right\rangle$ and $\langle\mathrm{F}\rangle$.

In this article we report in particular the high degree of the absorbency of the sodium salt of $\mathrm{CMC}(\mathrm{NaCMC})$, prepared from regenerated cellulose having the crystal form of cellulose II, toward various liquids and explain the absorbency on the basis of $\left\langle\left\langle\mathrm{f}_{k}\right\rangle\right.$ and $\langle\mathrm{F}\rangle$.

\section{EXPERIMENTAL}

\section{Synthesis of Carboxymethylcellulose}

As starting materials, four kinds of cellulose were used. Cellulose II (viscosity-average molecular weight $M_{v}=7.3 \times 10^{4}$ and degree of crystallinity by the X-ray diffraction method $\chi_{c}=46 \%$ ), regenerated from a cuprammonium cotton linter cellulose solution was used and is referred to as sample BLC. Natural wood pulp (cellulose I, $M_{v}=21 \times 10^{4}$ and $\chi_{\mathrm{c}}=76 \%$ ) and two acid-hydrolized wood pulps, prepared in $6 N$ sulfuric acid at $60^{\circ} \mathrm{C}$ for 15 and $65 \mathrm{~min}$ $\left(M_{v}=9.4 \times 10^{4}\right.$ and $\left.7.4 \times 10^{4}, \chi_{c}=77 \%\right)$ were used and are referred to as NC-1, NC-2, and $\mathrm{NC}-3$, respectively.

Twenty six samples of NaCMC having $\langle\mathrm{F}\rangle_{\text {Chem }}=0.01-0.64$, chemically determined, were synthesized in following manner: $20 \mathrm{~g}$ of cellulose were dipped in a $80 \mathrm{ml}$ of a system containing $3.3 \mathrm{~g}$ of sodium hydroxide and a mixture of 2-propanol-methanol-water $(56: 28: 16, \mathrm{v} / \mathrm{v} / \mathrm{v})$ at $25^{\circ} \mathrm{C}$ and allowed to stand for $30 \mathrm{~min}$. At $60^{\circ} \mathrm{C}$, 2-propanol solution saturated with monochloroacetic acid was added to the system. The reaction appeared heterogeneous and was processed at $60^{\circ} \mathrm{C}$ for $120 \mathrm{~min}$ without mechanical agitation. In this case, $0.07-7.15 \mathrm{~g}$ of monochloroacetic acid per $10 \mathrm{~g}$ of cellulose were added so as to obtain $\mathrm{CMC}$ with the desired $\langle\mathrm{F}\rangle$. Immediately on termination of the reaction, the resultant system was neutralized with a mixture of methanol-30 $\mathrm{wt} \%$ hydrochloric acid (9:1, $\mathrm{w} / \mathrm{w})$. The CMC in acid form was separated by filtration and washed with excess aq. me- thanol, immersed in a mixture of methanol$2 \mathrm{wt} \%$ aq. sodium chloride $(9: 1, \mathrm{w} / \mathrm{w})$ for $24 \mathrm{~h}$ for convertion into the salt form, washed again with aq. methanol, dried in air and then in vacuo at $70^{\circ} \mathrm{C}$ for $8 \mathrm{~h}$. By this method, 9 $\mathrm{NaCMC}$ samples, coded the BL series, were synthesized from BLC, 9 samples from NC-1 ( $\mathrm{N}-1$ series), 5 samples from NC-2 (N-2 series), and 3 samples from NC-3 (N-3 series).

\section{Viscosity-Average Molecular Weight}

The number-average and weight-average molecular weights of the NaCMC samples were not determined owing to experimental difficulty. We determined only $M_{v}$ of starting cellulose from the limiting viscosity number $[\eta]$ in cadoxen (cadomium oxide-sodium hydroxide-ethylenediamine-water $(4: 1: 12: 83$, $\mathrm{w} / \mathrm{w} / \mathrm{w} / \mathrm{w}$ ) using the Brown-Wikström relation $^{7}$ :

$$
[\eta]=3.85 \times 10^{-2} M_{w}^{0.76} \quad\left(\text { at } 25^{\circ} \mathrm{C}\right)
$$

\section{$X$-Ray Diffraction}

$\mathrm{X}$-Ray diffraction patterns of the original cellulose(s) and NaCMC were recorded with a RU-200PL type X-ray diffractometer (Rigaku Denki Co., Japan). $\chi_{c}$ of cellulose was calculated by the Segal's method, ${ }^{8}$ using the relation

$$
\chi_{\mathrm{c}}=\frac{I_{\mathrm{c}}-I_{\mathrm{a}}}{I_{\mathrm{c}}}
$$

Here, $I_{\mathrm{a}}$ and $I_{\mathrm{c}}$ are the relative intensities of the amorphous and crystalline peaks from the base line drawn by connecting the relative intensities at $2 \theta=4$ and $32^{\circ}$ (see Figure 1). For $\mathrm{NaCMC}, I_{\mathrm{c}}$ was defined as the highest intensity at the diffraction angle $2 \theta=19-30^{\circ}$ and $I_{\mathrm{a}}$, the lowest intensity at $2 \theta=15-20^{\circ}$.

\section{${ }^{13}$ C NMR Measurement}

Each of the four samples of the BL and N-1 series in a mixture of sodium hydroxidedeuterium oxide $(1: 9, \mathrm{w} / \mathrm{w})$ was measured for the ${ }^{13} \mathrm{C}$ NMR spectrum on a JEOL FX-400 and FX-500 pulse-Fourier Transform NMR 
Table I. Shielding and deshielding effects of the $O$-carboxymethyl group in 2-, 3-, and 6-substituted carboxymethyl glucose

\begin{tabular}{ccrrrrc}
\hline & \multicolumn{6}{c}{ Chemical shift from unsubstituted group } \\
\cline { 2 - 7 } Type of substitution & \multicolumn{1}{c}{$\mathrm{C}_{1}$} & $\mathrm{C}_{2}$ & \multicolumn{1}{c}{$\mathrm{C}_{3}$} & \multicolumn{1}{c}{$\mathrm{C}_{4}$} & \multicolumn{1}{c}{$\mathrm{C}_{5}$} & \multicolumn{1}{c}{$\mathrm{C}_{6}$} \\
\hline 2-Substituted & -0.4 & 8.1 & -1.2 & -0.4 & -0.3 & $-0.5--1.1$ \\
3-Substituted & -0.4 & -0.9 & 9.1 & -0.8 & -0.7 & $-0.9--1.0$ \\
6-Substituted & -0.3 & -0.7 & -0.8 & -1.1 & -1.8 & 8.1 \\
\hline
\end{tabular}

spectrometer $\left(100.7\right.$ and $125.9 \mathrm{MHz}$ for ${ }^{13} \mathrm{C}$ nuclei, respectively). The proton decoupled ${ }^{13} \mathrm{C} \mathrm{NMR}$ method in the NNE mode was applied using dioxane (67.8 ppm) as the internal standard. For detection of $\mathrm{CH}_{2}$ carbon peaks, the insensitive nuclei enhanced by the polarization transfer/complete decoupling (INEPT/COM) method $^{9}$ (delay time, $\Delta=$ $3(4 J)^{-1}, J$ being the scalar coupling constant between ${ }^{13} \mathrm{C}$ and ${ }^{1} \mathrm{H}$ ), was carried out.

\section{NMR Peak Assignment}

Using the reported results ${ }^{10,11}$ for $\beta$-glucose and its $\mathrm{C}_{2}-, \mathrm{C}_{3^{-}}$, and $\mathrm{C}_{6}$-monocarboxymethylated $\beta$-glucoses, the shielding or deshielding effect on ring carbons, induced by monosubstitution, was estimated (see Table I). By this results along with the ring carbon peaks of unsubstituted cellulose (i.e., $\mathrm{C}_{1}$, $104.7 ; \mathrm{C}_{2}, 75.0 ; \mathrm{C}_{3}, 76.4 ; \mathrm{C}_{4}, 80.0 ; \mathrm{C}_{5} ; 76.4$, and $\left.\mathrm{C}_{6}, 61.9 \mathrm{ppm}\right)$, the ring carbon peaks for all possible substituted forms of cellulose was calculated. For example, the peak position of the $\mathrm{C}_{1}$ carbon of $\mathrm{NaCMC}$ with $\left\langle\left\langle\mathrm{f}_{6}\right\rangle=1.00\right.$, $\left\langle\mathrm{f}_{2}\right\rangle=1.00, \quad$ and $\quad\left\langle\mathrm{f}_{3}\right\rangle=0.00, \quad$ is $104.7-0.4-0.3=104.0 \mathrm{ppm}$. The results are shown in Table II. The calculated peaks were compared with those observed.

\section{Determination of the Distribution of}

Substitution by the NMR Method

Total degree of substitution $\langle\mathrm{F}\rangle_{\mathrm{NMR}},\left\langle\left\langle\mathrm{f}_{6}\right\rangle\right.$, $\left\langle\mathrm{f}_{2}\right\rangle+\left\langle\left\langle\mathrm{f}_{3}\right\rangle\right.$ were calculated from ${ }^{13} \mathrm{C} N \mathrm{NMR}$ spectra using the following relations:

$$
\langle\mathrm{F}\rangle_{\mathrm{Chem}}=\frac{I_{\mathrm{CO}}}{I_{\mathrm{C}_{1}}}
$$

Polymer J., Vol. 17, No. 8, 1985

$$
\begin{gathered}
\left\langle\mathrm{f}_{6}\right\rangle=\frac{I_{\mathrm{SC}_{6}}}{I_{\mathrm{C}_{6}}+I_{\mathrm{SC}_{6}}} \\
\left\langle\mathrm{f}_{2}\right\rangle+\left\langle\mathrm{f}_{3}\right\rangle=\frac{I_{\mathrm{SC}_{2}}+I_{\mathrm{SC}_{3}}}{I_{\mathrm{C}_{1}}}
\end{gathered}
$$

where $I_{\mathrm{C}_{1}}, I_{\mathrm{CO}}, I_{\mathrm{C}_{6}}, I_{\mathrm{SC}_{6}}, I_{\mathrm{SC}_{2}}$, and $I_{\mathrm{SC}_{3}}$ are the integrated peak intensities of $\mathrm{C}_{1}$ carbon (103$105 \mathrm{ppm})$, CO carbon (180 ppm), unsubstituted $\mathrm{C}_{6}$ carbon $(61 \mathrm{ppm})$, substituted $\mathrm{C}_{6}$ carbon $(72.3 \mathrm{ppm})$ and substituted $\mathrm{C}_{2}$ and $\mathrm{C}_{3}$ carbons ( $81-86 \mathrm{ppm})$, respectively. Note that $\left\langle\mathrm{f}_{6}\right\rangle$ is independently determined from $\langle F\rangle_{\text {NMR }}$ or $\left\langle\mathrm{f}_{2}\right\rangle+\left\langle\left\langle\mathrm{f}_{3}\right\rangle\right.$ since the Overhauser effect in the $\mathrm{C}_{6}$ carbon was found somewhat smaller than in other carbons.

\section{Total Degree of Substitution Determined by Chemical Analysis}

Before determining $\langle\mathrm{F}\rangle_{\text {Chem }}$ by chemical analysis, $\mathrm{NaCMC}$ was converted to the acid form and immersed in a $3 \mathrm{wt}_{\mathrm{o}}^{\mathrm{O}}$ aq. sodium chloride solution. The hydrochloric acid thus produced was diluted with alkali and the excess of which was back-titrated with hydrochloric acid.

\section{Absorbency}

$W_{0}$ gram (about $0.5 \mathrm{~g}$ ) was placed in a nonwoven fabric bag having a weight of $a$ gram. The bag was immersed for $10 \mathrm{~min}$ at $37^{\circ} \mathrm{C}$ in pure water, $0.9 \mathrm{wt} \%$ of $\mathrm{NaCl}, \mathrm{CaCl}_{2}$, and $\mathrm{AlCl}_{3}$ aq. solutions, respectively. It was then suspended for $20 \mathrm{~min}$ in air to remove water adhering to the bag and sample and weighed $\left(\left(W_{1}+a\right)\right.$ gram $)$. The bag containing the 
NaCMC sample was dried and weighed $\left(\left(W_{2}+a\right)\right.$ gram). Absorbency $A$ and solubility $S$ were then defined by the following relations:

For water,

$$
\begin{aligned}
& A=\frac{W_{1}}{W_{2}} \times 100(\%) \\
& S=\frac{W_{0}-W_{2}}{W_{0}} \times 100(\%)
\end{aligned}
$$

and for aq. salt solutions

$$
\begin{aligned}
& A=\frac{W_{1}}{W_{2}-0.009\left(W_{0}-W_{1}\right)} \times 100(\%) \\
& S=\frac{W_{0}-W_{2}+0.009\left(W_{0}-W_{1}\right)}{W_{0}} \times 100(\%)
\end{aligned}
$$

Here, $W_{0}, W_{2}$, and $a$ were determined for sample which was conditioned at $20^{\circ} \mathrm{C}$ at $65 \%$ relative humidity for $24 \mathrm{~h}$.

\section{RESULTS AND DISCUSSION}

Figure 1 shows the change in X-ray diffraction patterns of cellulose I(A) and II(B) by alkali treatment and carboxymethylation. The characteristic diffraction peaks for both cellulose I $\left(2 \theta=9.0,14.7,16.4\right.$, and $\left.22.6^{\circ}\right)$ and II $\left(2 \theta=9.5,12.0,20.0\right.$, and $\left.21.5^{\circ}\right)$ remain distinct despite any treatment, although the X-ray diffraction pattern of $\mathrm{NaCMC}$ with relatively high $\langle\mathrm{F}\rangle(0.5-0.6)$ shows only the main peaks of the original celluloses. The original celluloses are thus not converted to alkali celluloses and the reaction is principally heterogeneous. Carboxymethylation first occurs in the amorphous region and proceeds to the crystalline part. Thus, the reaction from start to finish is influenced by the structure of the original celluloses.

Figure 2 shows $\chi_{\mathrm{c}}$ plotted against $\langle\mathrm{F}\rangle_{\mathrm{Chem}} \cdot \chi_{\mathrm{c}}$ of the NaCMC sample prepared from cellulose I decreases gradually as $\langle\mathrm{F}\rangle$ increases and abruptly drops to $10 \%$ at $\langle\mathrm{F}\rangle \simeq 0.4-0.5$. The hydroxyl group present in the crystalline region does not react with

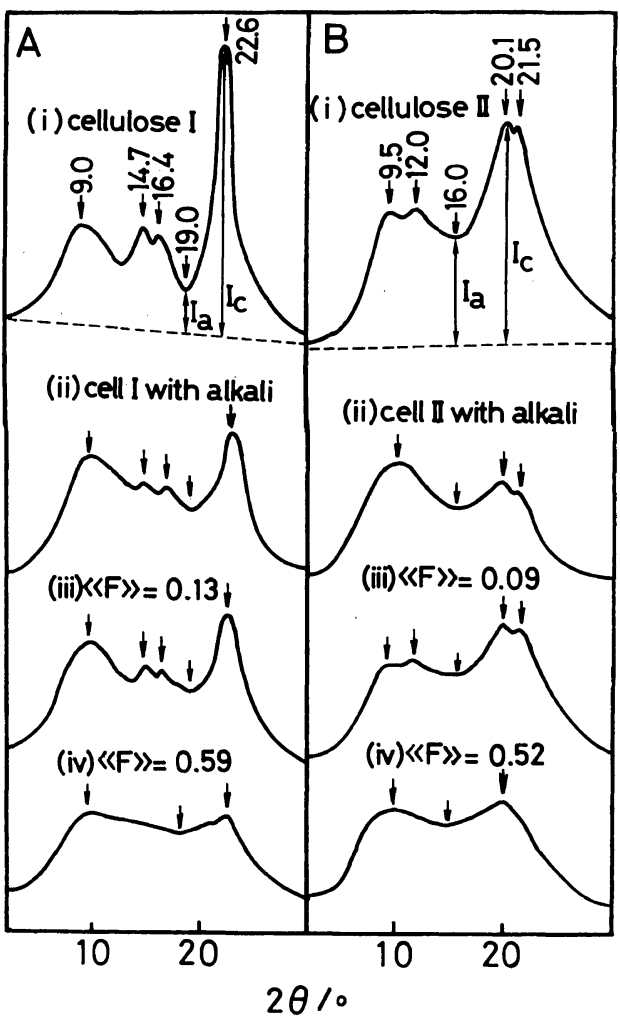

Figure 1. Change in X-ray diffraction curves of cellulose I (A) and II (B) alkali treatment and carboxymethylation: (A) (i), cellulose I; (ii), cellulose I with alkali; (iii), CMC $\left(\langle F\rangle_{\text {Chem }}=0.13\right)$ from cellulose I, (iv), CMC $\left(\langle\mathrm{F}\rangle_{\text {Chem }}=0.59\right)$ from cellulose I; (B) (I), cellulose II; (ii), cellulose II with alkali; (iii), $\mathrm{CMC}\left(\langle\mathrm{F}\rangle_{\mathrm{Chem}}=\right.$ $0.09)$ from cellulose II; (iv), CMC $\left(\langle\mathrm{F}\rangle_{\text {Chem }}=0.52\right)$ from cellulose II, $I_{\mathrm{a}}$ and $I_{\mathrm{c}}$ denote the relative intensity from amorphous and crystalline regions.

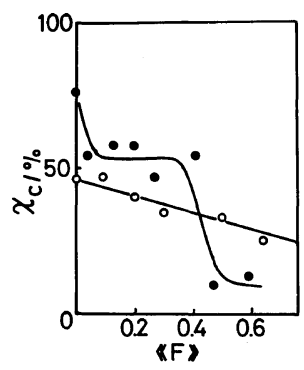

Figure 2. Relationships between crystallinity $\chi_{c}$ and total degree of substitution $\left\langle\langle\mathrm{F}\rangle_{\mathrm{Chem}}\right.$ : $\mathrm{O}, \mathrm{CMC}$ from cellulose II (BL series); $0, \mathrm{CMC}$ from cellulose I (N-1 series). 
Distribution of Substitution and Absorbency of CMC

Table II. Calculated chemical shift of CMC

\begin{tabular}{lcccccc}
\hline & \multicolumn{5}{c}{ Chemical shift $\delta /$ ppm } \\
\cline { 2 - 7 } Type of CMC & $\mathrm{C}_{1}$ & $\mathrm{C}_{2}$ & $\mathrm{C}_{3}$ & $\mathrm{C}_{4}$ & $\mathrm{C}_{5}$ & $\mathrm{C}_{6}$ \\
\hline Unsubstituted & 104.7 & 75.0 & 76.4 & 80.0 & 76.4 & 61.9 \\
2-Substituted & 104.3 & 85.9 & 75.2 & 79.6 & 76.1 & 61.4 \\
3-Substituted & 104.3 & 74.1 & 84.3 & 79.2 & 75.7 & 61.1 \\
6-Substituted & 104.4 & 74.3 & 75.6 & 78.9 & 74.6 & 70.0 \\
2,3-Substituted & 103.9 & 83.0 & 85.3 & 78.8 & 75.5 & 60.6 \\
2,6-Substituted & 104.0 & 83.1 & 74.4 & 78.5 & 74.3 & 69.5 \\
3,6-Substituted & 104.0 & 73.4 & 84.7 & 78.1 & 73.9 & 69.2 \\
2,3,6-Substituted & 103.6 & 82.3 & 83.5 & 77.7 & 73.6 & 68.7 \\
\hline
\end{tabular}

a $\mathrm{CH}_{2}$ for substituent group is expected to be around $71.2 \mathrm{ppm}$.

COONa for substituent group was seen at 178.4 and $178.8 \mathrm{ppm}$.

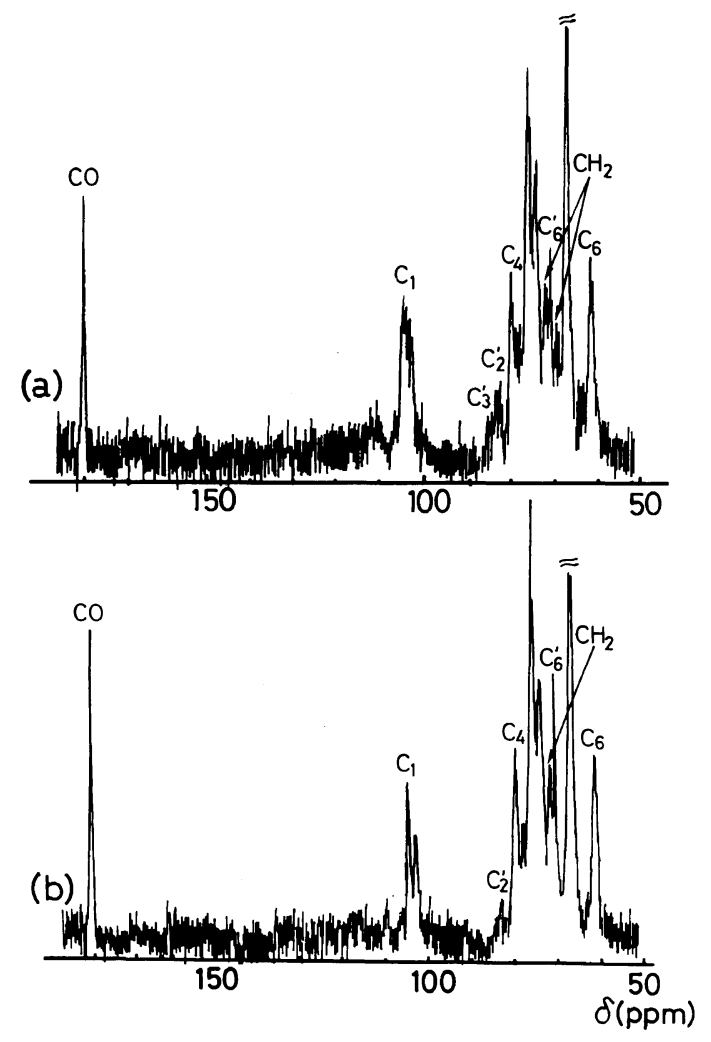

Figure 3. Typical ${ }^{13} \mathrm{C}$ NMR spectra of CMC (N-1-8) from cellulose I (a) and CMC (BL-9) from cellulose II (b).

monochloroacetic acid in a very random manner. $\chi_{c}$ of sample, prepared from cellulose II decreases linearly with increasing $\langle\langle F\rangle$. The cellulose II sample is a regenerated cellulose

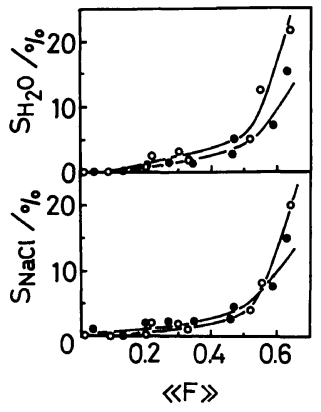

Figure 4. Solubility of $\mathrm{NaCMC}$ in $\mathrm{H}_{2} \mathrm{O}$ and $0.9 \mathrm{wt} \%$ aq. $\mathrm{NaCl}$ as a function of total degree of substitution $\langle\mathrm{F}\rangle_{\text {Chem }}$ : $O$, CMC from cellulose II (BL series), $\mathrm{CMC}$ from cellulose I ( $\mathrm{N}-1$ series).

having low $\chi_{c}$ and is very reactive with chemical reagents.

Figure 3 shows the ${ }^{13} \mathrm{C}$ NMR spectra of sample N-1-8 $\left(\langle\mathrm{F}\rangle_{\mathrm{Chem}}=0.59\right)$ and BL-9 $\left(\langle\mathrm{F}\rangle_{\text {Chem }}=0.64\right)$ at $125.7 \mathrm{MHz}$. The observed peak positions $\left(\mathrm{CO} ; 178.4-178.8 \mathrm{ppm}, \mathrm{C}_{1}\right.$; 103-104 ppm, substituted $\mathrm{C}_{2}$ and $\mathrm{C}_{3} ; 82-85$ ppm, substituted $\mathrm{C}_{6} ; 72.3 \mathrm{ppm}$, unsubstituted $\mathrm{C}_{6} ; 61.9 \mathrm{ppm}$ ) were in good agreement with those calculated in Table II and those reported by Parfondry et al. ${ }^{12}$ using commercial CMC decomposed enzymatically or by hydrolysis. Methylene carbons for carboxymethyl groups substituted at $\mathrm{C}_{2}$ and $\mathrm{C}_{6}$ are reported to be positioned at 67.6 and $72.6 \mathrm{ppm}$, respectively. But, in this study, the former peak was overlapped by a peak for the dioxane standard. 
K. KAMIDE et al.

Table III. Characterization and properties of CMC samples

\begin{tabular}{|c|c|c|c|c|c|c|c|c|c|c|c|c|}
\hline \multirow{2}{*}{ Sample } & \multirow{2}{*}{$\frac{M_{v}}{10^{4}}$} & \multicolumn{2}{|c|}{ Total $\langle F\rangle$} & \multicolumn{2}{|c|}{ Distribution } & \multicolumn{2}{|c|}{$S / \%$} & \multirow{2}{*}{$\frac{\chi_{\mathrm{c}}}{\%}$} & \multicolumn{4}{|c|}{$A / 100 \%$} \\
\hline & & $\begin{array}{l}\text { Chem. } \\
\text { anal. }\end{array}$ & NMR & $\left\langle\left\langle\mathrm{f}_{2}\right\rangle+\left\langle\left\langle\mathrm{f}_{3}\right\rangle\right.\right.$ & $\left\langle\mathrm{f}_{6}\right\rangle$ & $\mathrm{H}_{2} \mathrm{O}$ & $\mathrm{NaCl}$ & & $\mathrm{H}_{2} \mathrm{O}$ & $\mathrm{NaCl}$ & $\mathrm{CaCl}_{2}$ & $\mathrm{Al}_{2} \mathrm{Cl}_{3}$ \\
\hline BL-1 & 7.3 & 0.01 & - & - & - & 0.0 & 0.0 & - & 16.2 & 18.0 & - & - \\
\hline BL-2 & 7.3 & 0.09 & 0.03 & 0.00 & 0.03 & 0.0 & 0.0 & 46.9 & 17.5 & 17.5 & 13.5 & 15.2 \\
\hline BL-3 & 7.3 & 0.20 & 0.14 & 0.02 & 0.11 & 0.5 & 0.0 & 40.2 & 49.6 & 22.3 & 13.0 & 40.2 \\
\hline BL-4 & 7.3 & 0.22 & - & - & - & 2.5 & 2.0 & - & 51.9 & 25.0 & - & - \\
\hline BL-5 & 7.3 & 0.30 & 0.27 & 0.01 & 0.27 & 3.0 & 1.5 & 34.7 & 52.0 & 32.0 & 18.0 & 13.0 \\
\hline BL-6 & 7.3 & 0.33 & - & - & - & 2.0 & 1.0 & - & 60.0 & 38.5 & - & - \\
\hline BL-7 & 7.3 & 0.52 & - & - & - & 5.0 & 4.0 & 32.8 & 22.5 & 48.5 & - & - \\
\hline BL-8 & 7.3 & 0.55 & - & - & - & 12.5 & 8.0 & - & 20.0 & 45.0 & - & - \\
\hline BL-9 & 7.3 & 0.64 & 0.65 & 0.09 & 0.54 & 21.5 & 20.0 & 25.0 & 15.4 & 29.1 & 24.0 & 12.0 \\
\hline N-1-1 & 21.0 & 0.04 & - & - & - & 0.0 & 1.0 & 54.3 & 14.5 & 16.0 & - & - \\
\hline N-1-2 & 21.0 & 0.13 & - & - & - & 0.0 & 0.0 & 57.8 & 19.3 & 20.0 & - & - \\
\hline $\mathrm{N}-1-3$ & 21.0 & 0.20 & 0.24 & 0.16 & 0.08 & 1.0 & 2.0 & 57.8 & 29.1 & 22.0 & 13.5 & 13.7 \\
\hline $\mathrm{N}-1-4$ & 21.0 & 0.27 & - & - & - & 1.5 & 2.5 & 47.0 & 42.6 & 21.0 & - & - \\
\hline N-1-5 & 21.0 & 0.35 & 0.41 & 0.20 & 0.21 & 1.5 & 2.5 & 54.3 & 44.0 & 19.6 & 17.0 & 17.8 \\
\hline N-1-6 & 21.0 & 0.46 & - & - & - & 3.0 & 2.5 & - & 61.8 & 29.3 & - & - \\
\hline N-1-7 & 21.0 & 0.47 & - & - & - & 5.0 & 4.5 & 9.1 & 61.0 & 27.4 & - & - \\
\hline N-1-8 & 21.0 & 0.59 & 0.60 & 0.39 & 0.20 & 7.0 & 7.5 & 12.8 & 49.0 & 28.6 & 16.5 & 11.0 \\
\hline N-1-9 & 21.0 & 0.63 & 0.71 & 0.34 & 0.37 & 15.0 & 15.0 & - & 30.7 & 26.5 & 21.0 & 11.8 \\
\hline N-2-1 & 9.4 & 0.14 & - & - & - & - & - & - & 19.4 & 16.0 & - & - \\
\hline $\mathrm{N}-2-2$ & 9.4 & 0.24 & - & - & - & - & - & - & 29.0 & 15.5 & - & - \\
\hline $\mathrm{N}-2-3$ & 9.4 & 0.31 & - & - & - & - & - & - & 37.2 & 18.0 & - & - \\
\hline N-2-4 & 9.4 & 0.38 & - & - & - & - & - & - & 23.0 & 16.6 & - & - \\
\hline N-2-5 & 9.4 & 0.51 & - & - & - & - & - & - & 22.6 & 13.0 & - & - \\
\hline N-3-1 & 7.4 & 0.16 & - & - & - & - & - & - & 12.5 & 7.5 & - & - \\
\hline N-3-2 & 7.4 & 0.23 & - & - & - & - & - & - & 22.5 & 12.6 & - & - \\
\hline N-3-3 & 7.4 & 0.39 & - & - & - & - & - & - & 23.5 & 12.5 & - & - \\
\hline
\end{tabular}

Figure 4 indicates the peaks in region lower and higher than the substituted $\mathrm{C}_{6}$ carbon peak $(72.3 \mathrm{ppm})$ to be complicated in shape owing to incomplete proton decoupling, increasing their intensity in proportion to that of the substituted $\mathrm{C}_{2}$ and $\mathrm{C}_{3}$ carbon peaks (82-85 ppm). Their intensity for $\mathrm{N}-1-8$ is much stronger than those for BL-9, despite the same order of $\langle\langle\mathrm{F}\rangle$.

Table III shows the $M_{v},\left\langle\langle\mathrm{~F}\rangle_{\mathrm{Chem}},\langle\mathrm{F}\rangle_{\mathrm{NMR}}\right.$, $\left\langle\mathrm{f}_{k}\right\rangle$, solubility $S, \chi_{\mathrm{c}}$, and absorbency $A$ for the $\mathrm{NaCMC}$ samples. It should be noted that $\left\langle\langle\mathrm{F}\rangle_{\mathrm{NMR}}\right.$ coincided well with $\left\langle\langle\mathrm{F}\rangle_{\text {Chem }}\right.$ with an experimental uncertainty less than 0.04 and $\langle\mathrm{F}\rangle_{\mathrm{NMR}},\left\langle\left\langle\mathrm{f}_{2}\right\rangle+\left\langle\left\langle\mathrm{f}_{3}\right\rangle\right.\right.$, and $\left\langle\left\langle\mathrm{f}_{6}\right\rangle\right.$ evaluated independently satisfied the conditions $\langle\mathrm{F}\rangle_{\mathrm{NMR}}=$ $\left\langle\mathrm{f}_{2}\right\rangle+\left\langle\left\langle\mathrm{f}_{3}\right\rangle+\left\langle\mathrm{f}_{6}\right\rangle\right.$. This shows the equations used for evaluating the degree of substitution to be valid.

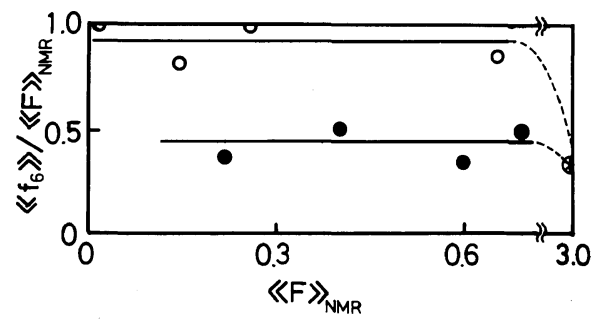

Figure 5. Plot of $\left\langle\left\langle\mathrm{f}_{6}\right\rangle /\left\langle\langle\mathrm{F}\rangle_{\mathrm{NMR}}\right.\right.$ versus $\langle\mathrm{F}\rangle_{\mathrm{NMR}}$. Symbols are the same as in Figure 4.

The solubility $S$ in water and aq. $\mathrm{NaCl}$ is plotted as a function of $\langle\vec{F}\rangle_{\text {Chem }}$ in Figure 4. $\mathrm{NaCMC}$ did not dissolve significantly in either aq. $\mathrm{CaCl}_{2}$ or aq. $\mathrm{AlCl}_{3} . S$ in water and in aq. $\mathrm{NaCl}$ was always less than $5 \%$ for $\langle\mathrm{F}\rangle_{\mathrm{Chem}}<0.55$ and increased remarkably when $\left\langle\langle\mathrm{F}\rangle_{\text {Chem }}\right.$ exceeded 0.55 .

Figure 5 shows a plot of the ratio $\left\langle\mathrm{f}_{6}\right\rangle \mid$ 


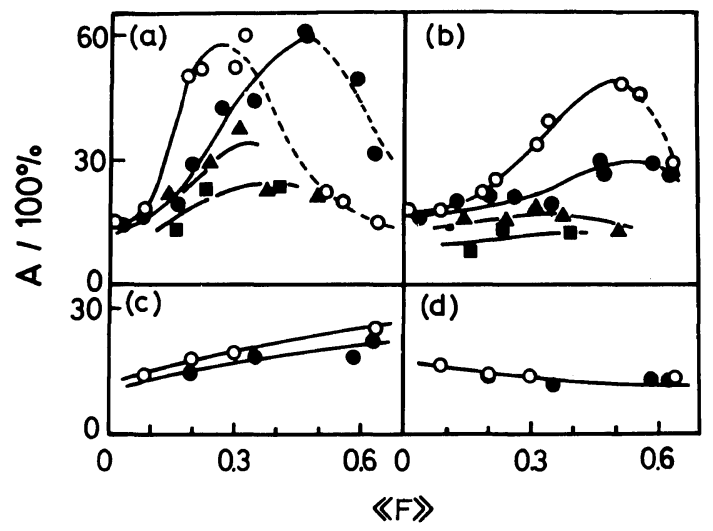

Figure 6. Absorbency $A$ of $\mathrm{NaCMC}$ toward various liquids as a function of total degree of substitution $\langle\mathrm{F}\rangle_{\text {chem }}$. a, $\mathrm{H}_{2} \mathrm{O} ; \mathrm{b}, 0.9 \mathrm{wt} \%$ aq. $\mathrm{NaCl} ; \mathrm{c}, 0.9 \mathrm{wt} \%$ aq. $\mathrm{CaCl}_{2} ; \mathrm{d}, 0.9 \mathrm{wt} \%$ aq. $\mathrm{AlCl}_{3} ; \mathrm{O}, \mathrm{CMC}$ from cellulose II (BL series, $\left.M_{v}=7.3 \times 10^{4}\right) ; 0, \mathrm{CMC}$ from cellulose I $(\mathrm{N}$ 1 series, $\left.M_{v}=21.0 \times 10^{4}\right) ; \boldsymbol{\Delta}, \mathrm{CMC}$ from cellulose I $(\mathrm{N}-2$ series, $\left.M_{v}=9.4 \times 10^{4}\right) ; \boldsymbol{\square}, \mathrm{CMC}$ from cellulose I $(\mathrm{N}-3$ series, $M_{v}=7.4 \times 10^{4}$ ).

$\langle\bar{F}\rangle_{\mathrm{NMR}}$ versus $\langle\mathrm{F}\rangle_{\mathrm{NMR}}$ for the $\mathrm{BL}$ (open mark) and the N-1 (closed mark) series CMC. $\left\langle\mathrm{f}_{6}\right\rangle /\langle\mathrm{F}\rangle_{\mathrm{NMR}}$ for the $\mathrm{BL}$ series CMC was $0.9 \pm 0.1$, when $\langle\vec{F}\rangle_{\mathrm{NMR}}<0.7$, indicating carboxymethylation to occur almost preferentially at the hydroxyl group attached to the $\mathrm{C}_{6}$ carbon on using cellulose II. $\left\langle\left\langle\mathrm{f}_{6}\right\rangle /\left\langle\langle\mathrm{F}\rangle_{\mathrm{NMR}}\right.\right.$ for $\mathrm{N}-1$ series was $0.43 \pm 0.1$ within $\langle\mathrm{F}\rangle_{\mathrm{NMR}}<0.7$. The reactivity of the hydroxyl groups is thus seen to depend very significantly on the cellulose sample, particularly its crystalline form.

\section{Factors Influencing Absorbency of $\mathrm{NaCMC}$}

Figure 6 shows the relations between absorbency $A$ against pure water (a), aq. $\mathrm{NaCl}$ (b), aq. $\mathrm{CaCl}_{2}$ (c), and aq. $\mathrm{AlCl}_{3}$ (d) and $\langle\mathrm{F}\rangle_{\text {Chem }}$ for N-1 $\left(M_{v}=21 \times 10^{4}\right.$, closed circle $)$ and $\mathrm{BL}$ $\left(M_{v}=7.3 \times 10^{4}\right.$, open circle) series. Figure 6a and $\mathrm{b}$ include the data for N-2 $\left(M_{v}=9.4 \times 10^{4}\right.$, closed triangle) and N-3 $\left(M_{v}=7.4 \times 10^{4}\right.$, closed rectangle) series in order to elucidate the molecular weight dependence of $A$. For the $\mathrm{N}$ series, $A$ increases somewhat with an increase in $M_{v}$ at a given $\langle\mathrm{F}\rangle_{\text {Chem }}$. Compared with $\mathrm{BL}$ series at the same $M_{v}$ level, the absolute $A$ value for the $\mathrm{N}-3$ series in water and aq. $\mathrm{NaCl}$

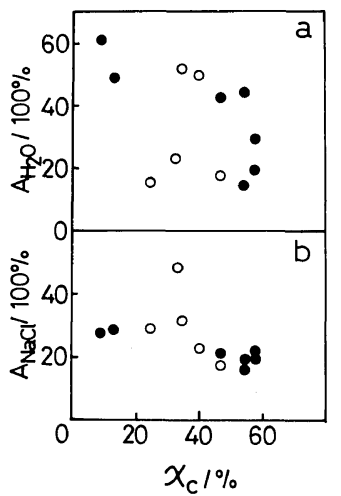

Figure 7. Absorbency $A$ of $\mathrm{NaCMC}$ towards $\mathrm{H}_{2} \mathrm{O}$ (a) and $0.9 \mathrm{wt} \%$ aq. $\mathrm{NaCl}$ (b) as a function of the degree of crystallinity $\chi_{\mathrm{c}}$. Symbols are the same as those in Figure 4.

is much lower than that of the BL series, indicating molecular weight not to be the main factor controlling $A$. On comparing the $\mathrm{BL}$ series with the $\mathrm{N}-1$ series, maximum $A$ in aq. $\mathrm{NaCl}$ of the former is 1.5 times that of the latter, so that $\mathrm{NaCMC}$ synthesized from cellulose II is particularly suitable as an absorbing agent, especially for aq. $\mathrm{NaCl}$ and similar solutions.

Neither $\left\langle\langle\mathrm{F}\rangle_{\mathrm{Chem}}\right.$ is the main factor since its value giving maximum $A$ against water and aq. $\mathrm{NaCl}$ differ according to the sample. $A$ in aq. $\mathrm{CaCl}_{2}$ increased gradually with an increase in $\left\langle\langle\mathrm{F}\rangle_{\text {Chem }}\right.$ for both samples. The $\mathrm{BL}$ series samples had a somewhat larger $A$ than that of the N-1 series. But, $A$ in aq. $\mathrm{AlCl}_{3}$ decreased slightly with an increase in $\left\langle\langle\mathrm{F}\rangle_{\mathrm{Chem}}\right.$, due of course to cross-linking between carboxyl groups and $\mathrm{Al}^{3+}$ cations.

Figure 7 shows the effects of the crystallinity $\chi_{\mathrm{c}}$ on $A$ in pure water (a) and in aq. $\mathrm{NaCl}(\mathrm{b})$. As $\chi_{\mathrm{c}}$ increased $A$ tended slightly to decrease, and the correlation coefficients $\gamma$ for $A$ and $\chi_{\mathrm{c}}$ were as follows: $A_{\mathrm{H}_{2} \mathrm{O}}-\chi_{\mathrm{c}},-0.34 ; A_{\mathrm{NaCl}}-\chi_{\mathrm{c}}$, -0.88 for the BL series and $A_{\mathrm{H}_{2} \mathrm{O}}-\chi_{\mathrm{c}},-0.79$ and $A_{\mathrm{NaCl}}-\chi_{\mathrm{c}},-0.82$ for the N-1 series. Thus, there is no significant correlation between $A$ and $\chi_{\mathrm{c}}$ and $\chi_{\mathrm{c}}$ is concluded not to be an additional factor controlling $A$. 


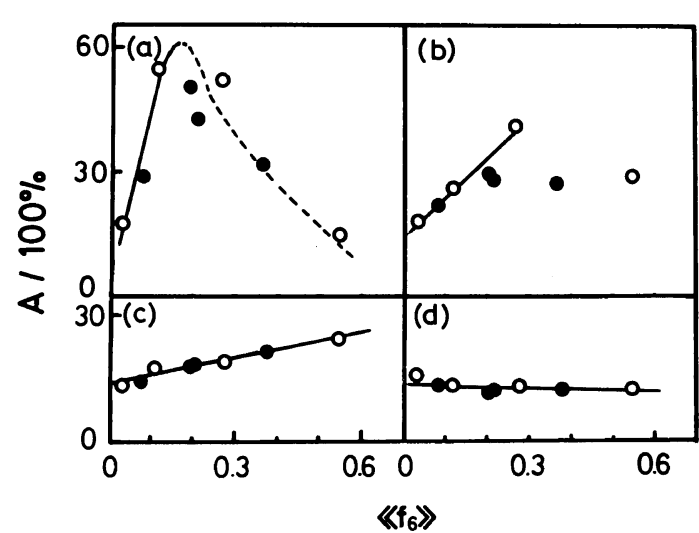

Figure 8. Absorbency $A$ of $\mathrm{NaCMC}$ towards various liquids as a function of $\left\langle\mathrm{f}_{6}\right\rangle: \mathrm{a}, \mathrm{H}_{2} \mathrm{O} ; \mathrm{b}, 0.9 \mathrm{wt} \%$ aq. $\mathrm{NaCl} ;$ c, $0.9 \mathrm{wt}^{\%} \%$ aq. $\mathrm{CaCl}_{2} ; \mathrm{d}, 0.9 \mathrm{wt} \%$ aq. $\mathrm{AlCl}_{3}$. Symbols are the same as those in Figure 4.

Figure 8 shows a plot of the absorbency $A$ against $\left\langle\mathrm{f}_{6}\right\rangle$, giving a single master curve for each liquid, irrespective of the sample series. As long as NaCMC does not dissolve in the liquid being used, the absorbency can be accurately determined by $\left\langle\mathrm{f}_{6}\right\rangle$. The following relations were obtained using the least-squares method:

$A=400\left\langle\mathrm{f}_{6}\right\rangle+7.0$ in pure water,

$$
\left\langle\mathrm{f}_{6}\right\rangle\langle 0.17
$$

$A=79\left\langle\mathrm{f}_{6}\right\rangle+14.2$ in $0.9 \mathrm{wt} \%$ aq. $\mathrm{NaCl}$,

$$
\left\langle\mathrm{f}_{6}\right\rangle<0.30
$$

$A=19.5\left\langle\left\langle\mathrm{f}_{6}\right\rangle+14.2\right.$ in $0.9 \mathrm{wt} \%$ aq. $\mathrm{CaCl}_{2}$,

$$
\left\langle\mathrm{f}_{6}\right\rangle<0.57
$$

$A=-3.25\left\langle\mathrm{f}_{6}\right\rangle+13.7$ in $0.9 \mathrm{wt} \%$ aq. $\mathrm{AlCl}_{3}$,

$$
\left\langle\mathrm{f}_{6}\right\rangle<0.57
$$

\section{Mechanisms of Carboxymethylation and Water Absorption by $\mathrm{NaCMC}$}

Figure 9 schematically illustrates the mechanism of carboxymethylation of cellulose $(\mathrm{a}-\mathrm{c})$ and that of water absorption by $\operatorname{NaCMC}\left(a \rightarrow a^{\prime}, b \rightarrow b^{\prime}, c \rightarrow c^{\prime}\right)$. In this figure, the full line is the cellulose chain and the

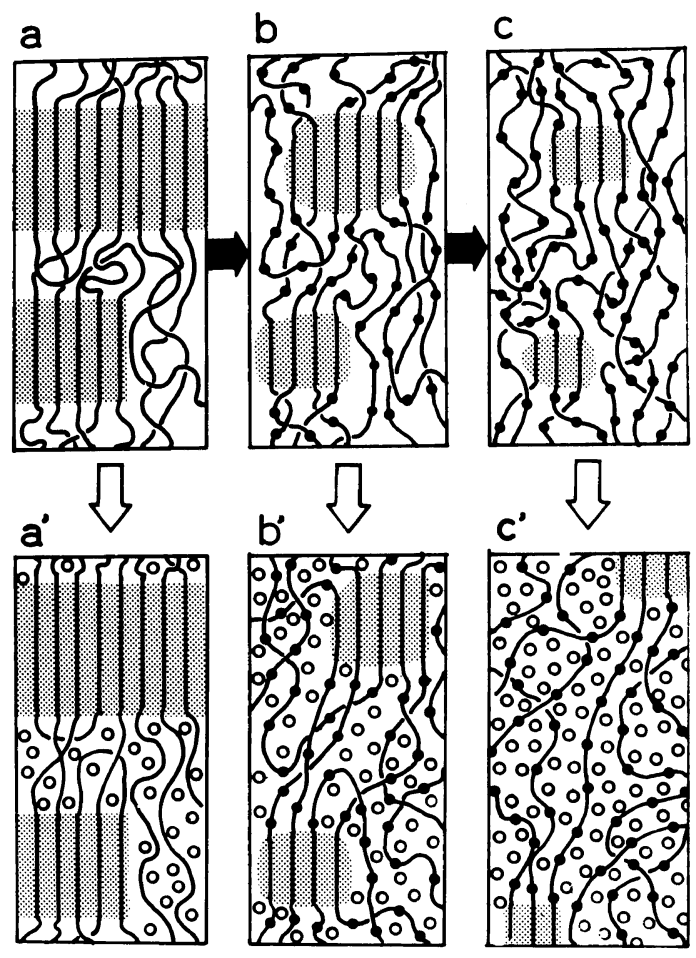

Figure 9. Schematic representation of carboxymethylation and swelling by water: —_, cellulose chain; - carboxymethylated glucose unit; $\mathrm{O}, \mathrm{H}_{2} \mathrm{O}$ molecule; shadowed area denotes crystalline part.

shadowed area, the crystalline region. The filled circle is carboxymethylated glucose and open circle, water molecules. On immersing cellulose (a) in water, only a part of the amorphous region absorbs water ( $\left.\mathrm{a}^{\prime}\right)$. Carboxymethylation of the cellulose occurs first in the amorphous region, the crystalline region collapses from the surface with further carboxymethylation (b and c). CMC with relatively low $\langle\mathrm{F}\rangle$ (b) dissociates, resulting in strong solvation with the surrounding water molecules and the consequent swelling $\left(b^{\prime}\right)$. $\mathrm{CMC}$ with relatively high $\langle\mathrm{F}\rangle(\mathrm{c})$ remains its smaller crystalline region and becomes hydrogel with its crystalline region as a kind of cross-linking point when immersed in water (c'). This mechanism is considered to be applicable to both $\mathrm{BL}$ and $\mathrm{N}$ series samples.

However, the reason for preferential car- 
boxymethylation at the $\mathrm{C}_{6}$ position of cellulose having the crystalline form of cellulose II and the paramount role of $\left\langle\mathrm{f}_{6}\right\rangle$ in the absorbency $A$ should be clarified in great detail. In considering the present carboxymethylation, it should be noted that 1) cellulose is not converted to alkali cellulose, 2) monochloroacetic acid reacts as an electrophilic reagent, ${ }^{12}$ and 3) carboxymethylation proceeds from an amorphous to crystalline region. A hydroxyl group having strong acidity in cellulose molecules thus reacts much more easily with monochloroacetic acid. This acidity is proportional for the most part to the electron density on its adjacent carbon atom. This density in turn governs the ${ }^{13} \mathrm{C}$ NMR resonance position, resonating at a higher magnetic field when the carbon has a higher electron density. A recent NMR analysis on cellulose showed the $C_{6}$ carbon NMR peak to appear at about $65-62$ ppm, while both $\mathrm{C}_{2}$ and $\mathrm{C}_{3}$ carbons resonate at $77-72 \mathrm{ppm}$, regardless of the crystalline form. The hydroxyl group attached to the $\mathrm{C}_{6}$ carbon thus reacts more easily with monochloroacetic acid than those at the $\mathrm{C}_{2}$ and $\mathrm{C}_{3}$ carbons. This agrees with the experimental facts that $\left\langle\mathrm{f}_{6}\right\rangle /$ $\langle\mathrm{F}\rangle_{\mathrm{NMR}}$ for celluloses I and II were found to be $0.43 \pm 0.1$ and $0.9 \pm 0.1$, respectively.

A more detailed investigation of the NMR spectrum of the $\mathrm{C}_{6}$ carbon region, carried out in our previous work ${ }^{13}$ indicated that 1) hydroxyl groups at the $\mathrm{C}_{6}$ position for cellulose II participate in two types of intramolecular hydrogen bonds $\left(\mathrm{O}_{2}-\mathrm{H} \cdots \mathrm{O}_{6}^{\prime}\right.$ and $\mathrm{O}_{6}-$ $\mathrm{H} \cdots \mathrm{O}_{2}^{\prime}$, the latter being much more acidic), 2) hydroxyl groups of cellulose I does not form the $\mathrm{O}_{6}-\mathrm{H} \cdots \mathrm{O}_{2}^{\prime}$ type intramolecular hydrogen bond, and 3) the electron density on $\mathrm{C}_{6}$ carbon for cellulose II in both ordered (crystalline) and amorphous regions is higher than that for cellulose $I$ and the reverse is true for the electron density on the $\mathrm{C}_{2}$ carbon. Thus, the reactivity of the hydroxyl group at the $\mathrm{C}_{6}$ position of cellulose II may possibly be relatively higher than that of cellulose I. The reverse situation applies at the $\mathrm{C}_{2}$ position.

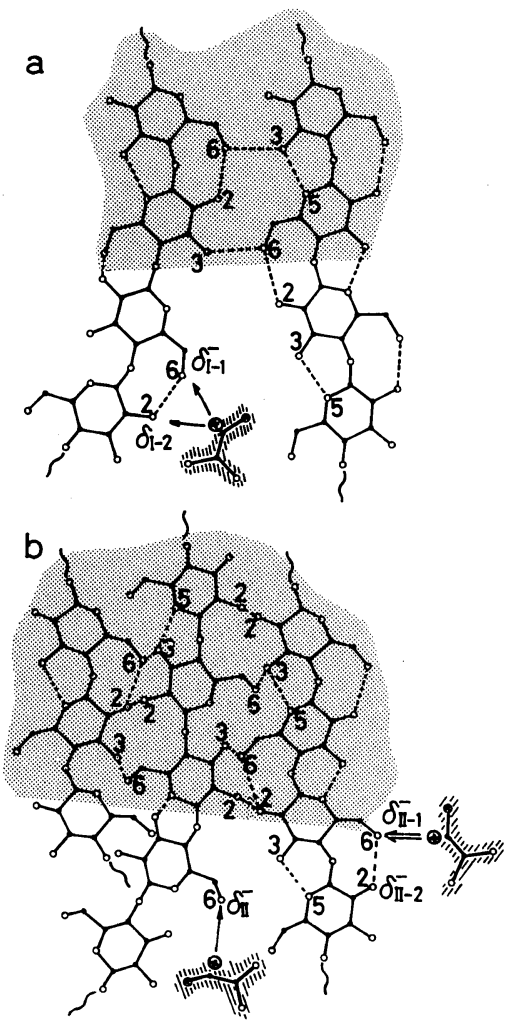

Figure 10. Schematic representation of carboxymethylation toward $\mathrm{O}_{2}-\mathrm{H} \cdots \mathrm{O}_{6}^{\prime}$ type intramolecular hydrogen bonds in cellulose I (a) and cellulose II (b). Shadowed area denotes the cationized monochloroacetic acid. $\delta_{\mathrm{I}-1}^{-}$ and $\delta_{\mathrm{I}-2}^{-}$mean the electronegativity $(\mathrm{EN})$ for cellulose I and $\delta_{\mathrm{II}}^{-}, \delta_{\mathrm{II}-1}^{-}$, and $\delta_{\mathrm{II}-2}^{-}, \mathrm{EN}$ for hydroxyl groups at the $\mathrm{C}_{6}$ and $\mathrm{C}_{2}$ positions of cellulose II. EN has the following order: $\delta_{\mathrm{II}-1}^{-}>\delta_{\mathrm{II}}^{-}>\delta_{\mathrm{I}-1}^{-}>\delta_{\mathrm{I}-2}^{-}>\delta_{\mathrm{II}-2}^{-}$. The $\mathrm{O}_{6}-\mathrm{H} \cdots \mathrm{O}_{2}^{\prime}$ type intramolecular hydrogen bonds for cellulose II are not shown. NE on the hydroxyl group at the $\mathrm{C}_{6}$ position is much stronger than $\delta_{\mathrm{I}-1}^{-}$.

The exclusively preferential substitution of the hydroxyl group at the $\mathrm{C}_{6}$ position does not occur in the case of cellulose I under the same conditions of carboxymethylation, as evident from Figure 10. The introduction of a bulky substituent at the $\mathrm{C}_{6}$ position destroys the intermolecular hydrogen bonds and widens the distance between molecular chains, creating a wider space to readily receive the absorbed liquids. This is because the hydroxyl groups at the $\mathrm{C}_{6}$ position in the original cellulose mainly govern the intermolecular hydrogen bonds. 


\section{K. KAMIDE $e t$ al.}

\section{REFERENCES}

1. E. Jansen, Ger. Patent, 332203 (1918).

2. see, for example, W. F. Waldeck and F. W. Smith, Ind. Eng. Chem., 44, 2803 (1952).

3. see, for example, R. H. Charles, US Patent 2607772 (1952).

4. C. D. Callihan, "Cellulose Technology Research," A. F. Turbak Ed., ACS Series 10, 1975, p 33.

5. E. Otto and H. M. Spurlin, "Cellulose and Cellulose Derivatives," Vol. II, John Wiley \& Sons Inc., New York, N. Y., 1954, p 944.

6. K. Kamide, K. Okajima, T. Matsui, M. Ohnishi, and H. Kobayashi, Polym. J., 13, 116 (1983).
7. W. Brown and R. Wikstrom, Eur. Polym. J., 1, 1 (1965).

8. L. Segal, Text. Res. J., 29, 786 (1959).

9. D. M. Doddrell and D. T. Pegg, J. Am. Chem. Soc., 102, 6388 (1980).

10. A. S. Perlin, B. Casu, and H. J. Koch, Can. J. Chem., 48, 2596 (1970).

11. A. Parfondry and A. S. Perlin, Carbohydr. Res., 57, 39 (1977).

12. see, for example, L. Fieser and M. Fieser, "Advanced Organic Chemistry," Reinhold Publishing Corp., New York, N. Y., 1961.

13. K. Kamide, K. Okajima, K. Kowsaka, and T. Matsui, Polym. J., 17, 701 (1985). 